A N N A L E S Annales de Bretagne et des Pays de l'Ouest

Anjou. Maine. Poitou-Charente. Touraine

$121-1 \mid 2014$

Varia

\title{
Louis Jacquinot, un indépendant en politique
}

\section{Christian Bougeard}

\section{OpenEdition}

Journals

Édition électronique

URL : http://journals.openedition.org/abpo/2762

DOI : $10.4000 / a b p o .2762$

ISBN : 978-2-7535-3407-0

ISSN : 2108-6443

Éditeur

Presses universitaires de Rennes

Édition imprimée

Date de publication : 27 mars 2014

Pagination : 206-209

ISBN : 978-2-7535-3405-6

ISSN : 0399-0826

Référence électronique

Christian Bougeard, «Louis Jacquinot, un indépendant en politique », Annales de Bretagne et des Pays de l'Ouest [En ligne], 121-1 | 2014, mis en ligne le 27 mars 2014, consulté le 22 septembre 2020. URL http://journals.openedition.org/abpo/2762 ; DOI : https://doi.org/10.4000/abpo.2762 
Histoire des prisonniers de guerre français, 1939-1945, Paris, FNCPG) ou, du même, de l'ouvrage publié dans la collection "La vie quotidienne " (La vie quotidienne des prisonniers de guerre dans les Stalags, les Oflags et les Kommandos, 1939-1945, Paris, Hachette 1987), aux études plus récentes de Fabien Théofilakis (Anne-Marie PATHE et Fabien TheOFILAKIS, dir., La captivité de guerre au XXe siècle. Des archives, des histoires, des mémoires, Paris, A. Colin, 2012; ou encore, chez l'éditeur des dessins de Garnier : Jean-Claude CATHERINE, dir., La captivité des prisonniers de guerre. Histoire et mémoire, 1939-1945, Rennes, Presses universitaire de Rennes, 2008) auraient permis de mieux mettre en valeur le corpus concernant François Garnier.

Les 25 dessins consacrés par le jeune artiste au Front-Stalag de Pithiviers, particulièrement rares, n'auraient-ils pas, par exemple, gagnés à être confrontés à ce que nous en disent les archives conservées dans le Loiret, émanant de la municipalité de la ville, de la préfecture ou de la presse locale? Plus qu'une notice générale sur les tirailleurs sénégalais (p. 33), dont l'exécution sommaire par les Allemands lors de la débâcle est évoquée par Garnier, n'aurait-il pas été préférable de rappeler ce que disent les travaux les plus récents sur ce point, des publications d'Armelle Mabon qui évoque notamment le cas de ces prisonniers noirs restés en Bretagne jusqu'en 1944 (Prisonniers de guerre indigènes. Visages oubliés de la France occupée, Paris, La Découverte, 2010) - à celles de Raffael Scheck (Une saison noire. Les massacres de tirailleurs sénégalais, mai-juin 1940, Paris, Tallandier, 2007)?

Et il en va de même de nombreux points, sur lesquels l'on aurait aimé que le lecteur soit éclairé quant à la banalité - ou pas - de ce que traduisent les dessins ou les lettres : du travail dans les Kommandos à la place du théâtre - bien mise en évidence dès l'entre-deux-guerres par la Grande Illusion, le film de Jean Renoir -, des relations avec les gardiens à la mise en récit par Garnier de ses hésitations face aux possibilités d'évasion qui se présentent à lui, pour n'en rester qu'à ces quelques exemples.

On l'aura compris : alors que de nouveaux médias permettent de saisir autrement l'expérience de la captivité entre 1940 et 1945 - l'on pense, entre autres, aux excellents albums BD de Jacques Tardi, Moi René Tardi, prisonnier de guerre au Stalag II B (Paris, Casterman, 2012), ou de Florent Silloray, Le carnet de Roger (Paris, Sarbacane, 2011), consacré dans ce dernier cas à son grand-père, un autre prisonnier breton, originaire de Nantes celui-là -, il nous semble que les dessins de François Garnier auraient mérité plus d'égards, plus encore dans une collection universitaire.

Yann LAGADEC

Bour, Julie, Gueit-Montchal, Lydiane, Dard, Olivier, Richard, Gilles (dir.), Louis Jacquinot, un indépendant en politique, Presses de l'université ParisSorbonne, 2013, 2008 p.

En dehors de la Meuse, département dont il a été député de 1932 à 1973 et président du Conseil général de 1945 à 1973, qui connaît encore Louis Jacquinot (1898-1993)? C'est pour rendre hommage à l'homme politique meusien mais aussi à l'ancien ministre sous trois Républiques qu'avec l'appui du Conseil général de la Meuse, les Archives départementales et le Centre de recherche universitaire lorrain d'histoire dirigé par Olivier Dard de l'université de Metz ont organisé en 2011 un colloque scientifique sur la carrière de Louis Jacquinot. Ce travail initié par Julie Bour a pu s'appuyer sur les fonds d'hommes politiques déposés aux archives départementales et présentés par leur directrice Lydiane Gueit-Montchal. En effet, ce fonds possède une très riche collection de photographies déposée par Louis Jacquinot 
dont les quatre cahiers publiés (50 pages de planches photographiques) font la richesse iconographique de l'ouvrage. En outre, loi du genre en histoire du temps présent, quatre hommes politiques ont apporté leur témoignage sur Jacquinot : un ancien député et un ancien sénateur de la Meuse ainsi que le sénateur Gérard Longuet alors ministre de la Défense. Celui d'Olivier Stirn, ancien chef de cabinet du ministre, est d'ailleurs assez décevant.

Dix communications permettent d'éclairer la longue carrière, le positionnement politique et l'action ministérielle de Louis Jacquinot qui fut brièvement sous-secrétaire d'État à l'Intérieur dans le cabinet Reynaud du 22 mars au 10 mai 1940, puis commissaire à la Marine du Comité Français de libération nationale (CFLN) à Alger et ministre au même poste en 1944-1945 et en 1947. Au total, Louis Jacquinot fut seize fois ministre indépendant (de droite) de la $\mathrm{III}^{\mathrm{e}}$ à la ve République, de 1940 à 1966. L'ouvrage est construit en trois parties : Louis Jacquinot en Meuse, sa carrière politique et son action ministérielle. Jean-Pierre Harbulot situe d'abord le notable de la droite modérée dans " l'environnement politique meusien " et une carrière qui s'inscrit dans le sillage des deux grands hommes politiques du département : Raymond Poincaré et André Maginot. Élu député de Commercy en 1932 par un véritable " coup de force " contre le député sortant du même parti politique Louis Taton-Vassal, pourtant soutenu par Poincaré, le jeune avocat installé à Paris (1926) dispose des réseaux locaux de son père, exploitant forestier, maire et conseiller d'arrondissement de Gondrecourt-le-Château. Mais il a surtout l'appui de l'autre homme fort, André Maginot, ministre de la Guerre et " père " de la célèbre ligne, qui le prend à son cabinet de 1928 à 1931. Le jeune député dispose du soutien des Meusiens de Paris où il vit en réalité, des Anciens combattants auquel il appartient et des milieux catholiques locaux. S'appuyant sur le monde rural, Louis Jacquinot va dominer la vie politique départementale jusqu'en 1973, date de son échec aux élections législatives lors de cette " élection de trop? " à laquelle il ne souhaitait pas se représenter. De ce point de vue, sa situation est comparable à celle de René Pleven, un homme politique de sa génération dont il avait été ministre entre 1950-1952. François Cochet précise quant à lui le parcours militaire de l'homme politique, futur ministre des Anciens combattants de la Ive République (octobre 1949-août 1951), présenté dans ses notices biographiques comme engagé volontaire en décembre 1916 et comme officier de liaison blessé par balle le 21 mai 1940 par un "tir ami ", ce qui lui vaut plusieurs décorations (Croix de guerre 1914-1918, Légion d'honneur le 6 décembre 1940). François Cochet montre que, comme bien d'autres, l'étudiant Jacquinot devance l'appel en 1916 par patriotisme sans doute mais aussi afin de choisir son arme, l'artillerie lourde beaucoup moins meurtrière que l'infanterie en première ligne. De même, du fait de sa blessure, en 1940 le député de Commercy est évacué de ville en ville pendant la débâcle jusqu'à Vichy où il se trouve le 10 juillet 1940, mais sur un lit d'hôpital, ce qui lui évite d'avoir à se prononcer sur les pleins pouvoirs au maréchal Pétain en ce jour où meurt la République.

Jean-François Murraciole examine les rapports entre Louis Jacquinot et la France libre. On sait peu de chose de son parcours de 1940 à janvier 1943, date de son évasion de France par l'Espagne réussie lors de sa deuxième tentative après deux arrestations (à Marseille en août et en tentant de franchir les Pyrénées en novembre 1942). Auparavant l'ancien député a intégré, sans doute à la fin 1941, le réseau de renseignement Alliance dépendant des Britanniques. Quant il arrive à Londres en mai 1943, comme certains parlementaires ralliés, Louis Jacquinot se méfie dans un premier temps du général de Gaulle, fréquentant même les cercles socialistes qui sont hostiles au Général. En pleine crise avec les giraudistes installés à Alger, les gaullistes se méfient eux aussi de cet homme de droite dont beaucoup de collègues sont à Vichy ou giraudistes. Mais à Londres, les anciens parlementaires de cette tendance ne sont pas 
si nombreux au moment où de Gaulle veut donner des gages de son républicanisme aux Américains. Avec Henri Queuille, Jacquinot gagne Alger le 10 août 1943 : il est sollicité aussi bien par de Gaulle que par Giraud mais il refuse plusieurs propositions car il attend du chef des Français libres des engagements républicains clairs dans le cadre d'une démocratie parlementaire rétablie. Tout change à l'automne 1943 quand il est désigné comme parlementaire à l'Assemblée consultative provisoire, puis nommé le 9 novembre commissaire à la Marine du CFLN, représentant des modérés dans ce comité élargi. Celui qui a alors la charge d'épurer la Marine et de fusionner ses différentes composantes dont les $9 / 10^{\mathrm{e}}$ ont été vichystes ou/et attentistes (à Alexandrie, aux Antilles) devient alors un gaulliste des plus fidèles.

Intérêt majeur de cet ouvrage, plusieurs études définissent le profil politique de cet " indépendant " dont la caractéristique constante reste la méfiance à l'égard des partis politiques et de leurs appareils et un positionnement souvent en marge, voire en opposition, avec les leaders des partis modérés auxquels il appartient. Ainsi, François Audigier démêle-t-il les relations de Louis Jacquinot avec l'Alliance démocratique $(\mathrm{AD})$, son parti d'origine qui a évolué du centre-droit vers la droite dans les années 1930. Le jeune avocat s'engage très à droite (proche des ligues) contre le Cartel des gauches en 1924-1926, suit son mentor Maginot à l'Alliance, mais une fois député, il est davantage un " compagnon de route " qu'un partisan engagé dans le fonctionnement du parti tout en se situant d'abord plutôt à la droite de cette formation. Proche de Paul Reynaud, il s'oppose en effet au leader de l'Alliance PierreEtienne Flandin. Il combat évidemment la politique du Front populaire mais pas de manière intransigeante votant certains textes sociaux. Surtout, Jacquinot refuse le virage néo-pacifiste, ce pacifisme d'abandon de Flandin face à la menace hitlérienne. De ce fait, il approuve la tentative de gouvernement d'union nationale de Léon Blum en mars 1938 (refusée par Flandin), et n'accepte pas les accords de Munich bruyamment applaudis par son chef de parti. Comme Paul Reynaud et Joseph Laniel, Jacquinot démissionne du groupe parlementaire de l'Alliance démocratique et en 1944 il s'oppose à la résurrection de ce parti. Gilles Richard montre qu'après guerre le " grand notable " Jacquinot a beaucoup de difficultés, de 1948 à 1962, à s'insérer dans le CNIP, le Centre national des Indépendants et Paysans, le grand parti des indépendants. Le ministre Louis Jacquinot est bien sûr réélu député de la Meuse en 1945 sur une liste alliée aux radicaux, mais il doit faire face à la concurrence du MRP et de la gauche (un député PCF en 1946). Par une politique d'alliance large, ce " libéral modéré " s'affirme comme le leader des droites locales sans adhérer pour autant au RPF qui présente une liste contre lui et obtient un député en 1951. Lors du regroupement des droites non-gaullistes au sein du CNIP en 1948, Louis Jacquinot rejoint cette formation dont il devient le principal ministre sous la IVe République, douze fois, plus qu'Antoine Pinay le leader du parti. Pourtant, pendant toute cette période, Jacquinot s'oppose presque constamment à la direction du CNIP (Pinay, Duchet; il vote contre la CED). Ce " grand notable rétif à une solide structure partisane " (Gilles Richard) est donc compatible avec le général de Gaulle qui en fait un de ses quatre ministres d'État quand il revient au pouvoir en juin 1958 pour créer un nouveau régime. Contrairement à de nombreux indépendants de droite, Jacquinot épouse et soutient la politique algérienne du général de Gaulle ainsi que la réforme constitutionnelle de 1962, ce qui provoque sa rupture avec le CNIP qui, en majorité, a voté la censure du gouvernement Pompidou. Il est vrai qu'il appartient à son gouvernement. Jérôme Pozzi éclaire d'ailleurs les liens particuliers de Louis Jacquinot avec le général de Gaulle et avec le gaullisme posant la question : un « indépendant gaulliste ou un gaulliste indépendant "? À partir de 1958, le ministre se rapproche de l'UNR sans le dire ouvertement se voulant toujours un "Républicain indépendant ". Mais après 1962, il ne rejoint pas le nouveau parti des Républicains indépendants de 
Valéry Giscard d'Estaing préférant s'apparenter au groupe parlementaire gaulliste en 1962 et 1967 avant de s'y inscrire en 1968. Il s'agit "d'un gaullisme de cœur " car Louis Jacquinot est en fait " un serviteur du gaullisme " qui préfère mener ses campagnes électorales à l'ancienne en s'appuyant sur les élus (maires, conseillers généraux) et ses réseaux locaux traditionnels et ruraux plutôt que sur des rassemblements trop politisés. Mais les mutations politiques, économiques et sociales à l'œuvre dans les années 1960-1970, dans la Meuse comme ailleurs, remettent en question cette façon de faire de la politique, et c'est ce qui explique son échec de 1973 alors que Georges Pompidou lui avait demandé de mettre le pied à l'étrier à son suppléant, Michel Drancourt, perçu par son électorat rural comme un " technocrate " parachuté. Dans cet ouvrage, la carrière ministérielle de Louis Jacquinot est précisée. Dans un long texte, Thomas Vaisset et Philippe Vial posent la question de savoir si Jacquinot aurait été le " Colbert de la Libération " pour répondre par la négative. Il n'en reste pas moins que celui qui a été le dernier ministre de la Marine de plein exercice a joué un rôle majeur pour refonder cette armée mise à mal par la guerre, à Alger d'abord, à Paris ensuite. Il s'est efforcé de restaurer cette fonction ministérielle fort discréditée par Vichy alors qu'il est surveillé de près par le général de Gaulle, " chef des armées ", et que ce ministère régalien est régulièrement divisé entre plusieurs hommes et plusieurs attributions de 1944 à 1948. Tout en épurant sérieusement la Marine, Jacquinot s'efforce de fusionner ses cadres très divisés par leur expérience de guerre, de réunifier ses différentes composantes et surtout de réhabiliter la Marine française après Mers-el Kébir et le sabordage de la flotte à Toulon. Actif défenseur des marins, Louis Jacquinot parvient à réarmer les navires engagés dans la guerre mais quand il veut reconstruire et moderniser l'outil naval, il se heurte dès 1945 et en 1947 aux réductions drastiques des crédits militaires, un peu relancés par la guerre d'Indochine. Malgré son opposition à Raul Ramadier en 1947, le ministre de la Marine ne peut entraver une mutation en cours achevée après 1958, à savoir l'intégration de la Marine dans un ensemble ministériel plus vaste.

Julie Bour étudie l'action de Louis Jacquinot, ministre des Anciens combattants dans quatre gouvernements de 1949 à 1951, qui au-delà des crises ministérielles assure une certaine continuité des politiques. Il s'efforce de défendre leurs intérêts et leurs pensions dans une période de guerre froide et d'amélioration de la situation économique du pays. Ensuite, dans des gouvernements de centre-droit et de droite, de 1951 à 1954, Louis Jacquinot occupe le ministère de la France d'OutreMer. Redevenu député, il s'oppose au processus d'indépendance de la Tunisie et du Maroc. Il appuie donc les guerres coloniales en Indochine et en Algérie. Sous la Ve République, il redevient le ministre d'État chargé des DOM et des TOM d'août 1961 à janvier 1966, c'est-à-dire qu'il est en charge de territoires réduits après la disparition de la " Plus grande France ». Frédéric Turpin analyse l'évolution d'un homme favorable à la colonisation française mais qui accepte les indépendances. Pendant cinq ans, sous la houlette du général de Gaulle, encadré par Jacques Foccart et aiguillonné par Michel Debré redevenu député de la Réunion, Louis Jacquinot s'efforce de promouvoir le développement économique et social dans les DOM-TOM pour conserver ces territoires à la France.

Dans leur conclusion, Olivier Dard et Gilles Richard synthétisent le parcours de ce notable modéré et républicain lorrain méconnu, formé dans la premier modèle républicain qui ne perçoit pas bien la recomposition du système partisan à l'œuvre dans les années 1968 même s'il avait parfaitement su prendre le virage de 1958 pour relancer sa carrière ministérielle. Alors que les biographies d'hommes politiques se multiplient, les éclairages croisés de cet ouvrage collectif sont donc les bienvenus. 